\title{
First-line pembrolizumab in programmed death ligand 1 positive non-small cell lung cancer
}

\author{
Camille Travert $^{1,2,3}$, Pascale Tomasini ${ }^{3}$, Arnaud Jeanson $^{3}$, Laurent Greillier $^{3}$, Fabrice Barlesi $^{3}$ \\ ${ }^{1}$ Pneumology and Oncology Department, Centre Hospitalo-Universitaire de Montpellier, Montpellier University, Montpellier, France; ${ }^{2}$ Department \\ of Pneumology and Oncology, Arnaud de Villeneuve hospital, Montpellier, France; ${ }^{3}$ Multidisciplinar Oncology \& Therapeutic Innovations \\ Department, Aix Marseille University, CNRS, INSERM, CRCM, APHM, Marseille, France \\ Correspondence to: Fabrice Barlesi. Sce Oncologie Multidisciplinaire \& Innovations Thérapeutiques, Hopital Nord, Chemin des Bourrely, 13015 \\ Marseille, France. Email: fabrice.barlesi@ap-hm.fr. \\ Comment on: Mok TSK, Wu YL, Kudaba I, et al. pembrolizumab versus chemotherapy for previously untreated, PD-L1-expressing, locally advanced \\ or metastatic non-small-cell lung cancer (KEYNOTE-042): a randomised, open-label, controlled, phase 3 trial. Lancet 2019;393:1819-30.
}

Submitted May 14, 2019. Accepted for publication May 19, 2019.

doi: $10.21037 /$ tcr.2019.05.20

View this article at: http://dx.doi.org/10.21037/tcr.2019.05.20

In these past few years, immune checkpoint inhibitors (ICI) in locally advanced or metastatic non-small cell lung cancer (NSCLC) have fundamentally changed the prognosis for some patients.

At the first, ICI were approved in pretreated locally advanced and metastatic NSCLC, first one nivolumab, independently of programmed death ligand 1 (PD-L1) status (1), rapidly followed by pembrolizumab for those with a PD-L1 expression of $1 \%$ or higher (2).

Regarding these extremely promising results, clinical trials in first line were designed, first ICI alone, and then treatment combination. The hope was to increase even more the treatment efficacy in first line, compared to second line.

Indeed, pembrolizumab was recently approved in first line for advanced or metastatic NSCLC patients presenting a $50 \%$ or higher expression of PD-L 1 . The median overall survival (OS) was 30 months (95\% CI, 18.3-NR months) versus 14.2 months, figures never seen before for tumors not harboring a molecular alteration leading to targeted therapies (3). Moreover, data indicate that pembrolizumab efficacy is linked to level of PD-L1 expression in a positive way (2).

Thus, KEYNOTE-042 (4) was designed to evaluate the efficacy of pembrolizumab in first line in patients suffering from a NSCLC and presenting a PD-L1 expression of $1 \%$ or higher. Cross-over was not allowed. One thousand two hundred seventy-four patients, mean age 63 years, were assigned to receive pembrolizumab $200 \mathrm{mg}$ every 3 weeks or platinum-doublet chemotherapy. Primary endpoint was OS in three different groups depending on PDL1 expression with a tumor proportion score (TPS) of 50\% or higher, $20 \%$ or higher and $1 \%$ or higher. Median OS in the pembrolizumab group versus chemotherapy group was better, with 20 months (95\% CI, 15.4-24.9 months) versus 12.2 months (95\% CI, 10.4-14.2 months), 17.7 months (95\% CI, 15.3-22.1 months) versus 13 months (95\% CI, 11.6-15.3 months) and 16.7 months (95\% CI, 13.919.7 months) versus 12 months (95\% CI, 11.3-13.3 months) respectively in the three populations. Pembrolizumab efficacy on OS improved with increasing PD-L1 TPS with hazard ratio (HR) of 0.69 (95\% CI, 0.56-0.85; $\mathrm{P}=0.0003$ ) for TPS $\geq 50 \%$, 0.77 (95\% CI, 0.64-0.92; $\mathrm{P}=0.0020$ ) for TPS $\geq 20 \%, 0.81$ (95\% CI, 0.71-0.93; $\mathrm{P}=0.0018$ ) for TPS $\geq 1 \%$. Pembrolizumab tolerance seemed to be the same as previously described. Mok et al. concluded that pembrolizumab could be extended to all NSCLC patients PD-L1 positive in first line.

Following these results, already presented at the annual meeting of the American Society of Clinical Oncology (ASCO) in June 2018, the Food and Drug Administration (FDA) recently approved pembrolizumab in first line for NSCLC patients with a PD-L1 status $\geq 1 \%$. It is expected that it will integrate the new guidelines of the American National Comprehensive Cancer Network (NCCN).

Thanks to this study, the benefit of pembrolizumab in first line for patients suffering from a NSCLC with a high PD-L1 expression $(\geq 50 \%)$ is confirmed. It also questions the place of pembrolizumab for all PD-L1 positive NSCLC patients.

But this study has some limits. First of all, the number of patients with PD-L1 TPS $\geq 50 \%$ was higher than previously reported (around 30\% usually) (3) and this could rise the effect of pembrolizumab.

Second, this OS analysis was hierarchical: the 
hypotheses were assessed sequentially. Thus, the benefit for the groups of TPS $\geq 20 \%$ and $\geq 1 \%$ could be explained by the benefit reached in the TPS $\geq 50 \%$ group. Indeed, in an exploratory analysis, there was no significant difference in OS between pembrolizumab and conventional chemotherapy for patients harboring a TPS between 1 and 49\% [OS 13.4 months (95\% CI, 10.718.2 months) versus 12.1 months (95\% CI, $11-14$ months)], with crossed survival curves (HR 0.92; 95\% CI, 0.77-1.11). This is consistent with the results of CheckMate 026 study. In this study nivolumab alone (an anti-PD-1) in first line for untreated NSCLC with a PD-L1 expression level of $5 \%$ or greater was not associated with a significantly longer OS than standard chemotherapy (14.4 months versus 13.2 months) (5). In another hand, BIRCH study, evaluating Atezolizumab in advanced NSCLC in a phase 2 trial, found an estimated OS of 23.5 months in first line (18.1-non estimated months) with an estimated OS of 26.9 months (12.0-non estimated months) in the group of patients with a PD-L1 expression of $50 \%$ or higher on tumor cells or of $10 \%$ or higher of tumor-infiltrating immune cells (6).

Third, results found for TPS $\geq 50 \%$ were different from KEYNOTE-024 study, with almost a 10 months difference, even cross-over was not allowed in KEYNOTE-042 study and only $20 \%$ of patients benefited from an anti-PD-1/PDL1 therapy after versus 64\% in KEYNOTE-024.

One reason could be the median number of cycles received. But it was not different between the two studies (9 for KEYNOTE-042 and 10.5 for KEYNOTE-024).

Another could be the type of population. There was a higher proportion of never smoker in KEYNOTE-042 than in KEYNOTE-024 (around 22\% in each group versus $3.2 \%$ in pembrolizumab group and $12.6 \%$ in chemotherapy group respectively).

Moreover, we have very few information about the genotypic alterations patients could present (other than EGFR mutation and ALK translocation which were exclusion criteria). As some molecular alterations are negative predictive factor of response and on the opposite, as tumor mutational burden appears to also be a predictive factor of response $(5,7)$, this data lack in the present study. Presence of other molecular drivers could explain the absence of benefit in the never-smoker subgroup seen, independently of PD-L1 status, especially since a third of the population came from east Asia.

In a statistical point of view, this study has also some bias. Primary objectives changed after the enrolment of 662 patients after KEYNOTE-010 results (2). This was previously designed with a primary objective of OS in TPS $\geq 50 \%$ group, they added OS in TPS $\geq 1 \%$ as a primary objective too. Two years after, after enrolment was complete, a third cut-off of TPS $\geq 20 \%$ was added, which was the second change in primary objective since the beginning of the study.

Fifth, there was an important number of screen fails: 2,153 over 3,428 patients $(62.8 \%)$, mostly because they did not respond to the inclusion and exclusion criteria, even these criteria were mostly common and did not differ from the other clinical trials evaluating ICI. The biggest reason why they were not included is a PD-L1 negative status, for 1,062 patients, which correspond to around $31 \%$ of the entire population. These figures are concordant with the literature $(5,8,9)$. Thus, these results correspond to a high selected population and may not be applicable in routine.

The place in first line of anti-PD1/PD-L1 therapy in NSCLC with an intermediate PD-L1 expression $\geq 1 \%$ but $<50 \%$ may be in combination. Several studies recently published are strongly in favor of combination with chemotherapy to sensitize NSCLC patients to ICI. KEYNOTE-407 and KEYNOTE-189 showed really encouraging results in OS both in squamous and non-squamous NSCLC, independently of PD-L1 status, with respectively an OS of 15.9 months in chemotherapy + pembrolizumab group versus 11.3 months in chemotherapy alone group in squamous NSCLC (10); and $69.2 \%$ of OS in combination group versus $49.4 \%$ in chemotherapy alone group at one year in non-squamous NSCLC (11). Atezolizumab, an anti-PD-L1 antibody, combined with chemotherapy in first line in non-squamous NSCLC demonstrated really promising results too, also independently from PD-L1 status, with an OS of 19.2 months in combination group versus 14.7 months in chemotherapy alone group (12).

ICI combinations of anti-PD-1/PD-L 1 and anticytotoxic T-lymphocyte antigen (CTLA)-4, as association of nivolumab and ipilimumab, showed also interesting in results in CheckMate 568, with a progression free survival of $42.6 \%$ versus $13.2 \%$ at one year in patients with a high $\mathrm{TMB}$, independently of PD-L1 status, but with a potential higher toxicity than chemotherapy combination (13).

In conclusion, KEYNOTE-042 is insufficient to allow pembrolizumab alone in first line in all PD-L1 positive NSCLC. European and French committees have not made any statement on its potentially approval yet. Despite its recent regulatory FDA approval, the best population which would benefit the most from pembrolizumab in first line has still to be elucidated, as the best strategy, including combination of ICI and/or chemotherapy. Pembrolizumab alone in first line could be proposed to patients who cannot receive a chemotherapy regimen because of a low general status or refusal.

\section{Acknowledgments}

Funding: None. 


\section{Footnote}

Provenance: and Peer Review: This article was commissioned and reviewed by the Section Editor Wei Xu (Division of Respiratory Disease, Department of Geriatrics, the First Affiliated Hospital of Nanjing Medical University, Nanjing, China).

Conflicts of Interest: All authors have completed the ICMJE uniform disclosure form (available at http://dx.doi. org/10.21037/tcr.2019.05.20). Dr. Barlesi reports personal fees from Astra-Zeneca, Bayer, Bristol-Myers Squibb, Boehringer-Ingelheim, Eli Lilly Oncology,F. HoffmannLa Roche Ltd, Novartis, Merck, MSD, Pierre Fabre, Pfizer and Takeda, outside the submitted work; Dr. GREILLIER reports personal fees and non-financial support from ABBVIE, personal fees and non-financial support from BRISTOL MYERS SQUIBB, personal fees and nonfinancial support from BOEHRINGER INGELHEIM, personal fees and non-financial support from ASTRA ZENECA, personal fees and non-financial support from ROCHE, personal fees and non-financial support from NOVARTIS, personal fees and non-financial support from MSD, personal fees and non-financial support from PFIZER, personal fees and non-financial support from TAKEDA,outside the submitted work; Dr. Tomasini reports grants, personal fees and non-financial support from Roche, grants, personal fees and non-financial support from AstraZeneca, personal fees and non-financial support from BMS, personal fees and non-financial support from Takeda,outside the submitted work; Dr. JEANSON has nothing to disclose; Dr. Travert has nothing to disclose.

Ethical Statement: The authors are accountable for all aspects of the work in ensuring that questions related to the accuracy or integrity of any part of the work are appropriately investigated and resolved.

Open Access Statement: This is an Open Access article distributed in accordance with the Creative Commons Attribution-NonCommercial-NoDerivs 4.0 International License (CC BY-NC-ND 4.0), which permits the noncommercial replication and distribution of the article with the strict proviso that no changes or edits are made and the original work is properly cited (including links to both the formal publication through the relevant DOI and the license). See: https://creativecommons.org/licenses/by-nc-nd/4.0/.

\section{References}

1. Borghaei H, Paz-Ares L, Horn L, et al. Nivolumab versus Docetaxel in Advanced Nonsquamous Non-Small-Cell Lung Cancer. N Engl J Med 2015;373:1627-39.
2. Herbst RS, Baas P, Kim DW, et al. Pembrolizumab versus docetaxel for previously treated, PD-L1-positive, advanced non-small-cell lung cancer (KEYNOTE-010): a randomised controlled trial. Lancet 2016;387:1540-50.

3. Reck M, Rodríguez-Abreu D, Robinson AG, et al. Pembrolizumab versus Chemotherapy for PD-L1Positive Non-Small-Cell Lung Cancer. N Engl J Med 2016;375:1823-33.

4. Mok TSK, Wu YL, Kudaba I, et al. Pembrolizumab versus chemotherapy for previously untreated, PD-L1expressing, locally advanced or metastatic non-small-cell lung cancer (KEYNOTE-042): a randomised, open-label, controlled, phase 3 trial. Lancet 2019;393:1819-30.

5. Carbone DP, Reck M, Paz-Ares L, et al. First-Line Nivolumab in Stage IV or Recurrent Non-Small-Cell Lung Cancer. N Engl J Med 2017;376:2415-26.

6. Peters S, Gettinger S, Johnson ML, et al. Phase II Trial of Atezolizumab As First-Line or Subsequent Therapy for Patients With Programmed Death-Ligand 1-Selected Advanced Non-Small-Cell Lung Cancer (BIRCH). J Clin Oncol 2017;35:2781-9.

7. Greillier L, Tomasini P, Barlesi F. The clinical utility of tumor mutational burden in non-small cell lung cancer. Transl Lung Cancer Res 2018;7:639-46.

8. Agarwal R, Dhooria S, Singh Sehgal I, et al. A Randomized Trial of Itraconazole vs Prednisolone in Acute-Stage Allergic Bronchopulmonary Aspergillosis Complicating Asthma. Chest 2018;153:656-64.

9. Dietel M, Savelov N, Salanova R, et al. Real-world prevalence of PD-L1 expression in locally advanced or metastatic nonsmall cell lung cancer (NSCLC): The global, multicentre EXPRESS study. J Thorac Oncol 2018;13:S74-5.

10. Paz-Ares L, Luft A, Vicente D, et al. Pembrolizumab plus Chemotherapy for Squamous Non-Small-Cell Lung Cancer. N Engl J Med 2018;379:2040-51.

11. Gandhi L, Rodríguez-Abreu D, Gadgeel S, et al. Pembrolizumab plus Chemotherapy in Metastatic NonSmall-Cell Lung Cancer. N Engl J Med 2018;378:2078-92.

12. Socinski MA, Jotte RM, Cappuzzo F, et al. Atezolizumab for First-Line Treatment of Metastatic Nonsquamous NSCLC. N Engl J Med 2018;378:2288-301.

13. Hellmann MD, Ciuleanu TE, Pluzanski A, et al. Nivolumab plus Ipilimumab in Lung Cancer with a High Tumor Mutational Burden. N Engl J Med 2018;378:2093-104.

Cite this article as: Travert C, Tomasini P, Jeanson A, Greillier L, Barlesi F. First-line pembrolizumab in programmed death ligand 1 positive non-small cell lung cancer. Transl Cancer Res 2019;8(7):2514-2516. doi: 10.21037/tcr.2019.05.20 